TRANSACTIONS OF THE

AMERICAN MATHEMATICAL SOCIETY

Volume 355, Number 3, Pages 1067-1077

S 0002-9947(02)03084-2

Article electronically published on October 24, 2002

\title{
EXPANDING MAPS ON INFRA-NILMANIFOLDS OF HOMOGENEOUS TYPE
}

\author{
KAREL DEKIMPE AND KYUNG BAI LEE
}

\begin{abstract}
In this paper we investigate expanding maps on infra-nilmanifolds. Such manifolds are obtained as a quotient $E \backslash L$, where $L$ is a connected and simply connected nilpotent Lie group and $E$ is a torsion-free uniform discrete subgroup of $L \rtimes C$, with $C$ a compact subgroup of $\operatorname{Aut}(L)$. We show that if the Lie algebra of $L$ is homogeneous (i.e., graded and generated by elements of degree 1), then the corresponding infra-nilmanifolds admit an expanding map. This is a generalization of the result of $\mathrm{H}$. Lee and K. B. Lee, who treated the 2-step nilpotent case.
\end{abstract}

\section{INTRODUCTION}

Let $L$ be a connected and simply connected nilpotent Lie group and let $C$ be a compact subgroup of Aut $(L)$, the group of continuous automorphisms of $L$. The group $L \rtimes \operatorname{Aut}(L)$, and therefore also $L \rtimes C$, acts on $L$ as follows:

$$
\forall m, n \in L, \forall \varphi \in \operatorname{Aut}(L):{ }^{(n, \varphi)} m=n \cdot \varphi(m) .
$$

A uniform discrete subgroup of $L \rtimes C$ is called an almost-crystallographic group. A torsion-free almost-crystallographic group is called an almost-Bieberbach group. If $E$ is an almost-Bieberbach group, then the quotient space $E \backslash L$ is a closed manifold, which is called an infra-nilmanifold.

If $E$ is an almost-Bieberbach group and if $\psi \in \operatorname{Aut}(L)$ is such that $\psi E \psi^{-1} \subseteq$ $E$, then $\psi$ induces a differentiable map on $E \backslash L$. Such a map is called an infranilmanifold endomorphism. Note that $\psi E \psi^{-1}$ can be a proper subgroup of $E$.

Let $M$ be a smooth closed manifold. A $C^{1}$-endomorphism $f: M \rightarrow M$ is said to be an expanding map if there exist constants $C>0$ and $\mu>1$ such that $\left\|D f^{n}(v)\right\| \geq C \mu^{n}\|v\|, \forall v \in T M$, for some Riemannian metric \|\| on $M$. In [3] it was shown that any expanding map of a compact manifold is topologically conjugate to an expanding infra-nilmanifold endomorphism. This is an infra-nilmanifold endomorphism which is induced by a Lie group automorphism all of whose eigenvalues (these are the eigenvalues of the corresponding automorphism on the Lie algebra) are of modulus $>1$.

In this paper, we will establish the existence of an expanding map for an elaborated class of infra-nilmanifolds, significantly generalizing the results obtained in 7 .

Received by the editors December 11, 2000 and, in revised form, March 15, 2002.

2000 Mathematics Subject Classification. Primary 37D20; Secondary 17B30, 17B70.

Key words and phrases. Infra-nilmanifold, expanding map, homogeneous Lie group. 
In fact, the class of infra-nilmanifolds we will consider is the class of infranilmanifolds of homogeneous type, which will be defined below. That this class is the natural class to investigate follows directly from the results of [5]. The main result of this paper is Theorem 5.1, stating that any infra-nilmanifold of homogeneous type admits an expanding map.

Let us begin by recalling a few basic facts about almost-Bieberbach groups. We refer to [1] for details about these results and for further references concerning almost-Bieberbach groups.

Let $E \subseteq L \rtimes C$ be an almost-crystallographic group. Then $N=E \cap L$ is a torsion-free finitely generated nilpotent group. Moreover $N$ is the Fitting subgroup of $E, N$ is maximal nilpotent in $E$ and $N$ is of finite index in $E$. The quotient group $F=E / N$ is called the holonomy group of $E$. Conversely, let

$$
1 \rightarrow N \rightarrow E \rightarrow F \rightarrow 1
$$

be an extension of groups with $F$ finite, $N$ finitely generated, torsion-free and maximal nilpotent in $E$. Then $E$ is isomorphic to an almost-crystallographic group. (We will say that $E$ is an abstract almost-crystallographic group.) The almostBieberbach groups share a rather strong uniqueness property:

Theorem 1.1 ([8]). Let $E_{1}, E_{2} \subseteq L \rtimes \operatorname{Aut}(L)$ be two almost-crystallographic groups. Then any isomorphism $\varphi: E_{1} \rightarrow E_{2}$ is induced by conjugation by an element of $L \rtimes \operatorname{Aut}(L)$.

This theorem implies that an infra-nilmanifold is completely determined by its fundamental group and that for our purposes we can always replace a given almostBieberbach group in $L \rtimes \operatorname{Aut}(L)$ by any of its isomorphic copies.

\section{Radicable hulls ANd Mal'CeV COMPletions}

In this section, we recall and apply the theory of canonical bases of torsion-free nilpotent groups. We refer the reader to [4] and [6, pp. 120ff] for the details.

Let $N$ be a finitely generated torsion-free nilpotent group. By refining the upper central series of $N$ we can find a central series

$$
N=N_{1} \supseteq N_{2} \supseteq N_{3} \supseteq N_{4} \supseteq \cdots \supseteq N_{d} \supseteq N_{d+1}=1
$$

with $N_{i} / N_{i+1} \cong \mathbb{Z}$, for each $i=1,2, \ldots, d$. It follows that it is possible to choose a generating set

$$
a_{1}, a_{2}, a_{3}, \ldots, a_{d}
$$

in such a way that $N_{i}$ is the group generated by $a_{i}$ and $N_{i+1}(\forall i=1,2, \ldots, d)$. We will refer to $\mathbf{a}=\left(a_{1}, a_{2}, \ldots, a_{d}\right)$ as a canonical basis of $N$. Given a canonical basis a of $N$, any element $n \in N$ is uniquely expressible as a product

$$
n=a_{1}^{z_{1}} a_{2}^{z_{2}} \cdots a_{d}^{z_{d}}, \text { with } z_{1}, z_{2}, \ldots, z_{d} \in \mathbb{Z}
$$

and we will call $\vec{z}=\left(z_{1}, z_{2}, \ldots, z_{d}\right)$ the canonical coordinates of $n$. Given $\vec{z} \in \mathbb{Z}^{d}$, we will use $n(\vec{z})$ to denote the element of $N$ whose canonical coordinates are $\vec{z}$. Canonical coordinates are interesting since products, morphisms, ... are expressed by means of polynomial maps.

Theorem 2.1. Let $N$ be a finitely generated torsion-free nilpotent group with a canonical basis $\mathbf{a}=\left(a_{1}, a_{2}, \ldots, a_{d}\right)$. Then: 
(1) There exists a polynomial function $\mu: \mathbb{Z}^{d} \times \mathbb{Z}^{d} \rightarrow \mathbb{Z}^{d}$ satisfying

$$
\forall \vec{x}, \vec{y} \in \mathbb{Z}^{d}: n(\vec{x}) \cdot n(\vec{y})=n(\mu(\vec{x}, \vec{y})) .
$$

(2) There exists a polynomial function $\lambda: \mathbb{Z} \times \mathbb{Z}^{d} \rightarrow \mathbb{Z}^{d}$ satisfying

$$
\forall k \in \mathbb{Z}, \forall \vec{x} \in \mathbb{Z}:(n(\vec{x}))^{k}=n(\lambda(k, \vec{x})) .
$$

(3) For any morphism $\varphi: N \rightarrow N$, there exists a polynomial function $\psi_{\varphi}$ : $\mathbb{Z}^{d} \rightarrow \mathbb{Z}^{d}$ satisfying

$$
\forall \vec{x} \in \mathbb{Z}^{d}: \varphi(n(\vec{x}))=n\left(\psi_{\varphi}(\vec{x})\right) .
$$

The polynomials in the theorem above are so-called "integer-valued" polynomials; these are polynomials with rational coefficients taking integer values when integers are substituted for the indeterminates. Using the theorem above, it is now possible to construct groups $N_{K}$ containing $N$. Let $K$ be any field of characteristic 0 . Then we define $N_{K}$ to be the set of all formal products of the form

$$
a_{1}^{k_{1}} a_{2}^{k_{2}} \cdots a_{d}^{k_{d}}, \quad k_{1}, k_{2}, \ldots, k_{d} \in K .
$$

As in the case of $N$, we denote this element by $n(\vec{k})$, where $\vec{k}=\left(k_{1}, k_{2}, \ldots, k_{d}\right)$. If one defines a product on $N_{K}$, using the same polynomial $\mu$ as in Theorem 2.1] one obtains a group which is torsion-free and nilpotent (of the same class as $N$ ). Moreover, any morphism of $N$ extends to a morphism of $N_{K}$ by using the same polynomial. In the special case $K=\mathbb{Q}$, the group $N_{\mathbb{Q}}$ is called the radicable hull (or rational Mal'cev completion or divisible closure) of $N$. Moreover, the extension of a morphism $\varphi$ of $N$ to $N_{\mathbb{Q}}$ is unique. In case $K=\mathbb{R}$, the group $N_{\mathbb{R}}$ is a Lie group, which is called the Mal'cev completion of $N$. This is the unique connected and simply connected nilpotent Lie group containing $N$ as a cocompact lattice. Also in this case, the extension of a morphism to a Lie group morphism (i.e., requiring continuity) of $N_{\mathbb{R}}$ is unique. Moreover, we can also uniquely extend any automorphism of $N_{\mathbb{Q}}$ to a Lie group automorphism of $N_{\mathbb{R}}$ (i.e., we have the following chain of groups: $\left.\operatorname{Aut}(N) \subseteq \operatorname{Aut}\left(N_{\mathbb{Q}}\right) \subseteq \operatorname{Aut}\left(N_{\mathbb{R}}\right)\right)$.

By mapping each element of $N_{\mathbb{Q}}$ (resp. $N_{\mathbb{R}}$ ) to its coordinate, we can identify (as sets) $N_{\mathbb{Q}}\left(\right.$ resp. $\left.N_{\mathbb{R}}\right)$ with $\mathbb{Q}^{d}$ (resp. $\left.\mathbb{R}^{d}\right)$. There is however a second way of making such an identification, using the associated Lie algebra.

Let $\mathfrak{n}_{\mathbb{Q}}$ denote the (rational) Lie algebra associated to $N_{\mathbb{Q}}$. It is well known that in the case of nilpotent groups, the exponential map

$$
\exp : \mathfrak{n}_{\mathbb{Q}} \rightarrow N_{\mathbb{Q}}
$$

is bijective and we can denote its inverse by log. (We refer the reader to [11] for a detailed treatment of this part of the section.) By choosing a basis $B_{1}, B_{2}, \ldots, B_{d}$ of $\mathfrak{n}_{\mathbb{Q}}$, we can identify $\mathfrak{n}_{\mathbb{Q}}$ with $\mathbb{Q}^{d}$, by mapping each element to its coordinate. Now, we find the second identification of $N_{\mathbb{Q}}$ with $\mathbb{Q}^{d}$ by mapping each element $n$ of $N_{\mathbb{Q}}$ onto the coordinate of $\log (n)$ with respect to the chosen basis. Lets us call this identification map $c_{\mathbb{Q}}$; thus

$$
c_{\mathbb{Q}}: N_{\mathbb{Q}} \rightarrow \mathbb{Q}^{d}: n \mapsto \text { coordinate of } \log (n) \text { w.r.t. a chosen basis. }
$$

Completely analogously, an identification $c o_{\mathbb{R}}$ of $N_{\mathbb{R}}$ with $\mathbb{R}^{d}$ can be obtained. The two identifications we described so far (canonical coordinates and Lie algebra coordinates) are polynomially related. We formulate this more precisely in the following theorem, which we only formulate for the radicable hull, but which also is valid for the Mal'cev completion. 
Theorem 2.2. Let $N$ be a torsion-free finitely generated group equipped with a canonical basis a and with radicable hull $N_{\mathbb{Q}}$. For a fixed basis $B_{1}, B_{2}, \ldots, B_{d}$ of $\mathfrak{n}_{\mathbb{Q}}$, there exist polynomial maps (with rational coefficients) $p_{1}, p_{2}: \mathbb{Q}^{d} \rightarrow \mathbb{Q}^{d}$, satisfying

(1) $\forall \vec{q} \in \mathbb{Q}^{d}: \operatorname{co}_{\mathbb{Q}}(n(\vec{q}))=p_{1}(\vec{q})$ and

(2) $\forall \vec{q} \in \mathbb{Q}^{d}: \operatorname{co}_{\mathbb{Q}}\left(n\left(p_{2}(\vec{q})\right)=\vec{q}\right.$.

For the proof of this theorem, we refer to [10, where the case of the (real) Mal'cev completion is handled (using the terminology of coordinates of the first/second kind).

Corollary 2.3. Let $N$ be a torsion-free finitely generated group equipped with a canonical basis a and with radicable hull $N_{\mathbb{Q}}$. For a fixed basis $B_{1}, B_{2}, \ldots, B_{d}$ of $\mathfrak{n}_{\mathbb{Q}}$ there exists a polynomial map $p: \mathbb{Q}^{d} \times \mathbb{Q}^{d} \rightarrow \mathbb{Q}^{d}$ satisfying

$$
c o_{\mathbb{Q}}\left(c o_{\mathbb{Q}}^{-1}\left(\vec{q}_{1}\right) \cdot c o_{\mathbb{Q}}^{-1}\left(\vec{q}_{2}\right)\right)=p\left(\vec{q}_{1}, \vec{q}_{2}\right) .
$$

Proof. This is just a combination of Theorem 2.1 and Theorem 2.2.

The following simple observation is crucial for the proof of the main result of this paper:

Proposition 2.4. Let $N$ be a torsion-free finitely generated group equipped with a canonical basis $\mathbf{a}$. Let $B_{1}, B_{2}, \ldots, B_{d}$ be a basis of the Lie algebra $\mathfrak{n}_{\mathbb{Q}}$ of the radicable hull $N_{\mathbb{Q}}$. Then there exists an integer $k \in \mathbb{Z}$ for which

(1) $\forall n \in N_{\mathbb{Q}}: c_{\mathbb{Q}}(n) \in k \mathbb{Z}^{d} \Rightarrow n \in N$

(2) $\forall n \in N: \operatorname{co}_{\mathbb{Q}}(n) \in \frac{1}{k} \mathbb{Z}^{d}$.

Proof. This is also a rather direct consequence of Theorem 2.2 Indeed, let $k_{1}$ be the least common multiple of the denominators of the coefficients of the map $p_{1}$. Then, if $\vec{q} \in \mathbb{Z}^{d}(\Leftrightarrow n(\vec{q}) \in N)$, then $\operatorname{co}_{\mathbb{Q}}(n(\vec{q})) \in \frac{1}{k_{1}} \mathbb{Z}^{d}$. Let $k_{2}$ be the least common multiple of the denominators of the coefficients of $p_{2}$. If one takes $\vec{q} \in k_{2} \mathbb{Z}^{d}$, then $p_{2}(\vec{q}) \in \mathbb{Z}^{d}$ (note that $p_{2}$ does not have constant terms!). This shows that if $c_{\mathbb{Q}}(n)=\vec{q} \in k_{2} \mathbb{Z}^{d}$, then $n \in N$. The lemma is proved by taking $k=k_{1} k_{2}$.

\section{Rational Realizations of ALmost-Bieberbach groups}

Let $E$ be an almost-Bieberbach group, with Fitting subgroup $N$. Then $E$ fits in a short exact sequence of the form

$$
1 \rightarrow N \rightarrow E \rightarrow F \rightarrow 1 .
$$

Since any automorphism of $N$ has a unique extension to $N_{\mathbb{Q}}$, we can form a commutative diagram

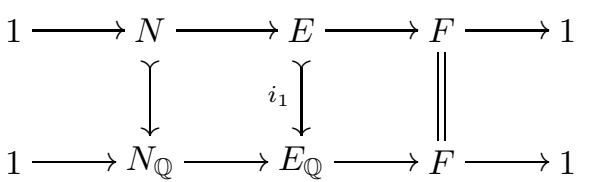

Lemma 3.1. The extension $1 \rightarrow N_{\mathbb{Q}} \rightarrow E_{\mathbb{Q}} \rightarrow F \rightarrow 1$ in diagram (3.1) is a split extension.

Proof. The proof of this lemma can be obtained as the rational analogue of [1] Lemma 3.1.2]. 
From now on, we fix a splitting $s: F \rightarrow E_{\mathbb{Q}}$ and we use $\varphi: F \rightarrow \operatorname{Aut}\left(N_{\mathbb{Q}}\right)$ to denote the induced morphism $\left(\varphi(f)(n)=s(f) n s(f)^{-1}\right)$. So $E_{\mathbb{Q}} \cong N_{\mathbb{Q}} \rtimes F$. We can also view (extend) each $\varphi(f)$ as an automorphism of $N_{\mathbb{R}}$ (we denote this by the same symbol), and so we can easily extend the diagram (3.1) above to obtain the following diagram:

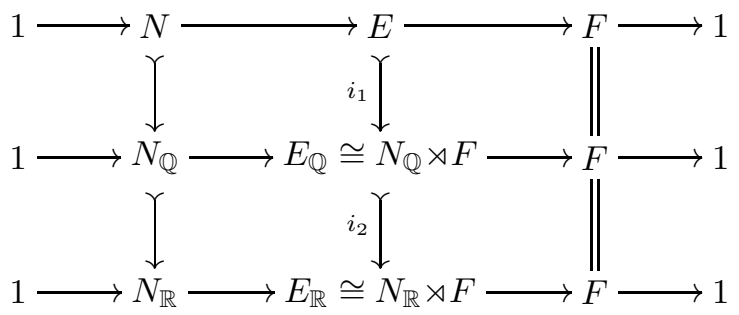

The embedding $i_{2} \circ i_{1}: E \rightarrow N_{\mathbb{R}} \rtimes F \subseteq N_{\mathbb{R}} \rtimes \operatorname{Aut}\left(N_{\mathbb{R}}\right)$ realizes $E$ as a genuine almost-Bieberbach group. Note that for all $e \in E$, we have that the image of $e$ is of the form $\left(n_{e}, \varphi\left(f_{e}\right)\right)$, where $f_{e}$ is the image of $e$ in $F$ (under the natural projection). Moreover, each $n_{e} \in N_{\mathbb{Q}}$, and therefore, it has rational canonical coordinates. We are also in the situation that $\varphi\left(f_{e}\right)$ is in fact an extended automorphism of $N_{\mathbb{Q}}$. Such realizations of an almost-Bieberbach group will be called rational realizations.

Definition 3.2. Let $E$ be an abstract almost-Bieberbach group with Fitting subgroup $N$. Assume $N$ is equipped with a canonical basis a leading to a radicable hull $N_{\mathbb{Q}}$. An embedding $i: E \rightarrow N_{\mathbb{Q}} \rtimes \operatorname{Aut}\left(N_{\mathbb{Q}}\right)$ that is the identity on $N$ will be called a rational realization of $E$.

Note that if $i: E \rightarrow N_{\mathbb{Q}} \rtimes \operatorname{Aut}\left(N_{\mathbb{Q}}\right)$ is a rational realization of $E$, then, the prolongation of this map to $N_{\mathbb{R}} \rtimes \operatorname{Aut}\left(N_{\mathbb{R}}\right)$ realizes $E$ as a genuine almost-Bieberbach group.

Above we have established the following lemma.

Lemma 3.3. Any almost-Bieberbach group admits a rational realization.

\section{LiE ALGEBRAS OF HOMOGENEOUS TYPE}

We will say that a finite-dimensional Lie algebra $\mathfrak{g}$ (over a given field) is of homogeneous type if it admits a subspace $\mathfrak{g}_{1}$ such that $\mathfrak{g}=\mathfrak{g}_{1} \oplus[\mathfrak{g}, \mathfrak{g}]$ and such that $\mathfrak{g}$ can be written as

$$
\mathfrak{g}=\mathfrak{g}_{1} \oplus \mathfrak{g}_{2} \oplus \mathfrak{g}_{3} \oplus \cdots,
$$

where $\mathfrak{g}_{i+1}=\left[\mathfrak{g}_{1}, \mathfrak{g}_{i}\right]$. This definition of homogeneous type coincides with the same concept of [5] and with the notion of quasi-cyclic of [9].

Note that, if a given Lie algebra $\mathfrak{g}$ can be written as a direct sum (4.1) with

(1) $\forall i, j>0:\left[\mathfrak{g}_{i}, \mathfrak{g}_{j}\right] \subseteq \mathfrak{g}_{i+j}$ (i.e., $\mathfrak{g}$ is graded) and

(2) $\mathfrak{g}$ is generated by the elements of $\mathfrak{g}_{1}$,

then $\mathfrak{g}$ is homogeneous. This follows from the fact that for such $\mathfrak{g}$ the lower central series, defined as $\gamma_{1}(\mathfrak{g})=\mathfrak{g}$ and $\gamma_{i+1}(\mathfrak{g})=\left[\mathfrak{g}, \gamma_{i}(\mathfrak{g})\right]$, is given by

$$
\gamma_{i}(\mathfrak{g})=\mathfrak{g}_{i} \oplus \mathfrak{g}_{i+1} \oplus \mathfrak{g}_{i+2} \oplus \cdots .
$$

Moreover, if $X_{1}, X_{2}, \ldots, X_{c}$ is a basis of $\mathfrak{g}_{1}$, then $\mathfrak{g}_{j}$ is generated by all $j$-fold brackets

$$
\left[X_{i_{1}}, X_{i_{2}}, \ldots, X_{i_{j}}\right] \text { with } 1 \leq i_{1}, i_{2}, \ldots, i_{j} \leq c
$$


A direct sum decomposition (4.1) of a Lie algebra of homogeneous type satisfying the conditions mentioned above will be called a grading of $\mathfrak{g}$. Note that we always assume that for a given grading, $\mathfrak{g}$ is generated by the elements of $\mathfrak{g}_{1}$ (i.e., the elements of homogeneous degree 1).

Note that any 2-step nilpotent Lie algebra is of homogeneous type, while there are 3-step nilpotent Lie algebras which are not of homogeneous type (and which do not allow an expanding automorphism). Of course, all finite-dimensional Lie algebras of homogeneous type are nilpotent.

We will also say that a Lie group is of homogeneous type if its Lie algebra is of homogeneous type. Also, an infra-nilmanifold or an almost-Bieberbach group is said to be of homogeneous type if the corresponding Lie group is.

Lemma 4.1. Let $\mathfrak{g}=\mathfrak{g}_{1} \oplus \mathfrak{g}_{2} \oplus \mathfrak{g}_{3} \oplus \cdots$ be a finite-dimensional Lie algebra (over any field) of homogeneous type. Assume that $Y_{1}, Y_{2}, \ldots, Y_{c} \in \mathfrak{g}$ are elements such that their canonical projections $\bar{Y}_{1}, \bar{Y}_{2}, \ldots, \bar{Y}_{c}$ form a basis of $\mathfrak{g} /\left(\mathfrak{g}_{2} \oplus \mathfrak{g}_{3} \oplus \mathfrak{g}_{4} \oplus \cdots\right)$. Then

$$
\mathfrak{g}=\mathfrak{h}_{1} \oplus \mathfrak{h}_{2} \oplus \mathfrak{h}_{3} \oplus \mathfrak{h}_{4} \oplus \cdots
$$

where $\forall i: \mathfrak{h}_{i}$ is the vector space spanned by all $i$-fold commutators

$$
\left[Y_{j_{1}}, Y_{j_{2}}, \ldots, Y_{j_{i}}\right], \quad 1 \leq j_{1}, j_{2}, \ldots, j_{i} \leq c .
$$

Moreover,

(1) $\left[\mathfrak{h}_{i}, \mathfrak{h}_{j}\right] \subseteq \mathfrak{h}_{i+j}$, and

(2) $\mathfrak{g}$ is generated by $\mathfrak{h}_{1}$.

Proof. Since $\mathfrak{g}$ is finite dimensional, there exists an $m$ with $\mathfrak{g}_{n}=0$ for $n>m$. Let us prove the lemma by induction on this $m$. If $m=1$, then $Y_{1}, Y_{2}, \ldots, Y_{c}$ form a basis of $\mathfrak{g}_{1}$, and so $\mathfrak{g}=\mathfrak{g}_{1}=\mathfrak{h}_{1}$. Now, assume that $m>1$. Note that $\mathfrak{h}_{n} \subseteq \gamma_{n}(\mathfrak{g})=\mathfrak{g}_{n} \oplus \mathfrak{g}_{n+1} \oplus \cdots ;$ therefore $\mathfrak{h}_{n}=0$ if $n>m$.

We will first show that $\mathfrak{h}_{m}=\mathfrak{g}_{m}$. The inclusion $\mathfrak{h}_{m} \subseteq \mathfrak{g}_{m}$ is obvious. By the hypothesis on the $Y_{i}$ 's, we can find coefficients $\alpha_{i, j}$ in the field we are working in, such that

$$
X_{i}=\sum_{j=1}^{c} \alpha_{i, j} Y_{j}+w_{i} \text { with } w_{i} \in \gamma_{2}(\mathfrak{g}) .
$$

Let $X=\left[X_{i_{1}}, X_{i_{2}}, \ldots, X_{i_{m}}\right]$ be an $m$-fold commutator. Then, we have that

$$
\begin{aligned}
X & =\left[X_{i_{1}}, X_{i_{2}}, \ldots, X_{i_{m}}\right] \\
& =\left[\sum_{j=1}^{c} \alpha_{i_{1}, j} Y_{j}+w_{i_{1}}, \sum_{j=1}^{c} \alpha_{i_{2}, j} Y_{j}+w_{i_{2}}, \ldots, \sum_{j=1}^{c} \alpha_{i_{m}, j} Y_{j}+w_{i_{m}}\right] \\
& =\left[\sum_{j=1}^{c} \alpha_{i_{1}, j} Y_{j}, \sum_{j=1}^{c} \alpha_{i_{2}, j} Y_{j}, \ldots, \sum_{j=1}^{c} \alpha_{i_{m}, j} Y_{j}\right] \\
& =\sum_{1 \leq j_{1}, j_{2}, \ldots, j_{m} \leq c} \alpha_{i_{1}, j_{1}} \alpha_{i_{2}, j_{2}} \cdots \alpha_{i_{m}, j_{m}}\left[Y_{j_{1}}, Y_{j_{2}}, \ldots, Y_{j_{m}}\right] \in \mathfrak{h}_{m},
\end{aligned}
$$


showing that $\mathfrak{g}_{m} \subseteq \mathfrak{h}_{m}$, as desired. The inclusion map $i: \mathfrak{h} \rightarrow \mathfrak{g}$ induces a commutative diagram

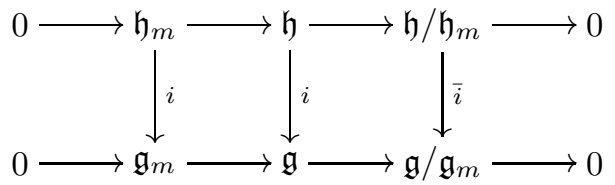

By the induction hypothesis, we know that $\bar{i}$ is an isomorphism. It follows that also the middle arrow $i$ is an isomorphism, which concludes the proof.

Corollary 4.2. Let $N$ be a finitely generated torsion-free nilpotent group equipped with a canonical basis $\mathbf{a}$. Then $\mathfrak{n}_{\mathbb{Q}}$ is of homogeneous type iff $\mathfrak{n}_{\mathbb{R}}$ is of homogeneous type.

Proof. Let $\mathfrak{n}_{\mathbb{Q}}$ be of homogeneous type. Then we can take a grading

$$
\mathfrak{n}_{\mathbb{Q}}=\mathfrak{n}_{\mathbb{Q}, 1} \oplus \mathfrak{n}_{\mathbb{Q}, 2} \oplus \mathfrak{n}_{\mathbb{Q}, 3} \oplus \cdots
$$

We now find the desired grading of $\mathfrak{n}_{\mathbb{R}}=\mathfrak{n}_{\mathbb{Q}} \otimes_{\mathbb{Q}} \mathbb{R}$ by taking

$$
\mathfrak{n}_{\mathbb{R}}=\left(\mathfrak{n}_{\mathbb{Q}, 1} \otimes_{\mathbb{Q}} \mathbb{R}\right) \oplus\left(\mathfrak{n}_{\mathbb{Q}, 2} \otimes_{\mathbb{Q}} \mathbb{R}\right) \oplus\left(\mathfrak{n}_{\mathbb{Q}, 3} \otimes_{\mathbb{Q}} \mathbb{R}\right) \oplus \cdots
$$

Now, suppose that $\mathfrak{n}_{\mathbb{R}}$ is of homogeneous type and that

$$
\mathfrak{n}_{\mathbb{R}}=\mathfrak{n}_{\mathbb{R}, 1} \oplus \mathfrak{n}_{\mathbb{R}, 2} \oplus \mathfrak{n}_{\mathbb{R}, 3} \oplus \cdots
$$

is a grading for $\mathfrak{n}_{\mathbb{R}}$. Now choose (rational) vectors $Y_{1}, Y_{2}, \ldots, Y_{c} \in \mathfrak{n}_{\mathbb{Q}}$ in such a way that their natural projections form a basis of $\mathfrak{n}_{\mathbb{R}} / \gamma_{2}\left(\mathfrak{n}_{\mathbb{R}}\right)$. By the lemma above, such a choise of basis induces a grading

$$
\mathfrak{n}_{\mathbb{R}}=\mathfrak{h}_{1} \oplus \mathfrak{h}_{2} \oplus \mathfrak{h}_{3} \oplus \cdots .
$$

However, by taking only rational linear combinations of the vectors $Y_{1}, Y_{2}, \ldots, Y_{c}$ and their $i$-fold brackets $(i=1,2, \ldots)$, we find a grading of $\mathfrak{n}_{\mathbb{Q}}$.

As a consequence, we have that an infra-nilmanifold is of homogeneous type if the corresponding Lie algebra $\mathfrak{n}_{\mathbb{Q}}$ is of homogeneous type.

Proposition 4.3. Let $\mathfrak{g}$ be a finite-dimensional Lie algebra of homogeneous type over any field $K$ of characteristic 0 . Let $F \subseteq \operatorname{Aut}(\mathfrak{g})$ be a finite group of automorphisms of $\mathfrak{g}$. Then there exists a grading

$$
\mathfrak{g}=\mathfrak{g}_{1} \oplus \mathfrak{g}_{2} \oplus \mathfrak{g}_{3} \oplus \cdots
$$

of $\mathfrak{g}$ such that $\forall \varphi \in F: \varphi\left(\mathfrak{g}_{i}\right)=\mathfrak{g}_{i}$

Proof. The proof of this proposition is similar to that of Proposition 5.1 of [2]. Let

$$
\mathfrak{g}=\mathfrak{h}_{1} \oplus \mathfrak{h}_{2} \oplus \mathfrak{h}_{3} \oplus \cdots
$$

be any grading of $\mathfrak{g}$. Fix a basis $X_{1}, X_{2}, \ldots, X_{c}$ of $\mathfrak{h}_{1}$ and complement it with a basis $Z_{1}, Z_{2}, \ldots, Z_{m}$ of $\mathfrak{h}_{2} \oplus \mathfrak{h}_{3} \oplus \cdots$ to obtain a basis of $\mathfrak{g}$. With respect to this basis, each element $\varphi \in F$ is expressed by a matrix of the form

$$
\varphi \approx\left(\begin{array}{cc}
A(\varphi) & 0 \\
N(\varphi) & B(\varphi)
\end{array}\right)
$$

where $A(\varphi) \in \mathrm{GL}(c, K), B(\varphi) \in \mathrm{GL}(m, K)$ and $N(\varphi)$ is an $m \times c$ matrix $\left(\in K^{m \times c}\right)$. Let

$$
\lambda: F \rightarrow K^{m \times c}: \varphi \mapsto N(\varphi) A(\varphi)^{-1} .
$$


We claim that $\lambda$ is a 1 -cocycle with respect to the $F$-module structure of $K^{m \times c}$ given by

$$
\forall \varphi \in F, \forall M \in K^{m \times c}:{ }^{\varphi} M=B(\varphi) M A\left(\varphi^{-1}\right) .
$$

Indeed, an easy calculation shows that

$$
\begin{aligned}
\lambda(\varphi \psi) & =N(\varphi \psi) A\left(\psi^{-1}\right) A\left(\varphi^{-1}\right) \\
& =(B(\varphi) N(\psi)+N(\varphi) A(\psi)) A\left(\psi^{-1}\right) A\left(\varphi^{-1}\right) \\
& ={ }^{\varphi} \lambda(\psi)+\lambda(\varphi),
\end{aligned}
$$

and the claim holds. As $F$ is finite and $K^{m \times c}$ is a divisible $F$-module, we know that $H^{1}\left(F, K^{m \times c}\right)=0$. It follows that there is a matrix $C \in K^{m \times c}$ such that $\forall \varphi \in F: \lambda(\varphi)={ }^{\varphi} C-C$. Now, we define a change of basis given by the matrix

$$
\left(\begin{array}{cc}
I_{c} & 0 \\
-C & I_{m}
\end{array}\right)
$$

(i.e., we do not change the $Z_{i}$, but we only alter the $X_{i}$ with an $\left(\mathfrak{h}_{2} \oplus \mathfrak{h}_{3} \oplus \cdots\right)$-part). Let us call this new basis $Y_{1}, Y_{2}, \ldots, Y_{c}, Z_{1}, Z_{2}, \ldots, Z_{m}$. With respect to the new basis the element $\varphi$ is represented by a matrix

$$
\begin{aligned}
\left(\begin{array}{cc}
I_{c} & 0 \\
C & I_{m}
\end{array}\right)\left(\begin{array}{cc}
A(\varphi) & 0 \\
\lambda(\varphi) A(\varphi) & B(\varphi)
\end{array}\right)\left(\begin{array}{cc}
I_{c} & 0 \\
-C & I_{m}
\end{array}\right) \\
=\left(\begin{array}{cc}
A(\varphi) & 0 \\
C A(\varphi)+\lambda(\varphi) A(\varphi)-B(\varphi) C & B(\varphi)
\end{array}\right) \\
=\left(\begin{array}{cc}
A(\varphi) & 0 \\
0 & B(\varphi)
\end{array}\right) .
\end{aligned}
$$

Let $\mathfrak{g}_{1}$ be the space spanned by $Y_{1}, Y_{2}, \ldots, Y_{c}$. The computation above shows that $\varphi\left(\mathfrak{g}_{1}\right)=\mathfrak{g}_{1}$. Moreover, by taking $\mathfrak{g}_{i}$ to be the space spanned by all $i$-fold Lie brackets in the basis vectors $Y_{1}, Y_{2}, \ldots, Y_{c}$, we find a grading

$$
\mathfrak{g}=\mathfrak{g}_{1} \oplus \mathfrak{g}_{2} \oplus \mathfrak{g}_{3} \oplus \mathfrak{g}_{4} \cdots
$$

satisfying $\varphi\left(\mathfrak{g}_{i}\right)=\mathfrak{g}_{i}, \forall \varphi \in F$.

\section{EXPANDING MAPS}

This section is devoted to the proof of the main result of this paper:

Theorem 5.1. Let $M$ be an infra-nilmanifold of homogeneous type. Then $M$ admits an expanding map.

Assume that the infra-nilmanifold $M$ is determined by the almost-Bieberbach group $E$. As usual, we denote the Fitting subgroup of $E$ by $N$ and the holonomy group by $F$. We fix a canonical basis a of $N$ (section 2) and also a rational realization

$$
i: E \rightarrow N_{\mathbb{Q}} \rtimes \operatorname{Aut}\left(N_{\mathbb{Q}}\right) \quad(\operatorname{section} 3) .
$$

Using this rational realization and after having fixed a splitting for $1 \rightarrow N_{\mathbb{Q}} \rightarrow$ $E_{\mathbb{Q}} \rightarrow F \rightarrow 1$ as in section [3, we can view $F$ as a finite subgroup of $\operatorname{Aut}\left(N_{\mathbb{Q}}\right)=$ $\operatorname{Aut}\left(\mathfrak{n}_{\mathbb{Q}}\right)$.

As it was assumed that $E$ is of homogeneous type, we can choose a grading (Proposition 4.3)

$$
\mathfrak{n}_{\mathbb{Q}}=\mathfrak{n}_{1} \oplus \mathfrak{n}_{2} \oplus \mathfrak{n}_{3} \oplus \mathfrak{n}_{4} \cdots
$$


with

$$
\forall \varphi \in F: \quad \varphi\left(\mathfrak{n}_{i}\right)=\mathfrak{n}_{i}, \quad i=1,2,3, \ldots
$$

For any $\lambda \in \mathbb{Q} \backslash\{0\}$, we will use $K_{\lambda}$ to denote the automorphism of $N_{\mathbb{Q}}$ (and also of $N_{\mathbb{R}}$ ) which, on the Lie algebra level, corresponds to the automorphism $k_{\lambda}$, where $k_{\lambda}$ restricted to $\mathfrak{n}_{i}$ is multiplication by $\lambda^{i}, i=1,2,3,4, \ldots$. (The reader can easily check that such a $k_{\lambda}$ is indeed an automorphism of $\mathfrak{n}_{\mathbb{Q}}$.)

As $k_{\lambda}$ restricted to $\mathfrak{n}_{i}$ is just multiplication by a scalar, we have that $K_{\lambda}$ commutes with all elements of $F$. Using $K_{\lambda}$, we can define an automorphism $\tilde{K}_{\lambda}$ of $E_{\mathbb{Q}}=N_{\mathbb{Q}} \rtimes F$ as follows:

$$
\tilde{K}_{\lambda}: N_{\mathbb{Q}} \rtimes F \rightarrow N_{\mathbb{Q}} \rtimes F:(n, \varphi) \mapsto\left(K_{\lambda}(n), \varphi\right) .
$$

It is our intention to show that $\tilde{K}_{\lambda}(i(E)) \subseteq i(E)$ for a good choice of $\lambda>1$.

We formulate this in the following lemma.

Lemma 5.2. Using the notation introduced above, we can find an integer $\lambda>1$ satisfying

$$
\forall e \in i(E): \tilde{K}_{\lambda}(e) \cdot e^{-1} \in i(E) .
$$

Proof. Fix a basis $B_{1}, B_{2}, \ldots, B_{d}$ of $\mathfrak{n}_{\mathbb{Q}}$, leading to an identification $c o \mathbb{Q}$. By Proposition 2.4 there exists a $k \in \mathbb{Z}$ satisfying

(1) $\forall n \in N_{\mathbb{Q}}: \operatorname{co}_{\mathbb{Q}}(n) \in k \mathbb{Z}^{d} \Rightarrow n \in N$, and

(2) $\forall n \in N: \operatorname{co}_{\mathbb{Q}}(n) \in \frac{1}{k} \mathbb{Z}^{d}$.

Now, let $e \in i(E) \subseteq N_{\mathbb{Q}} \rtimes F$. Then we can view $e$ as being a couple $e=\left(n_{e}, \varphi_{e}\right)$, with $n_{e} \in N_{\mathbb{Q}}$ and $\varphi_{e} \in F$.

The proof of the lemma makes use of 4 facts.

Fact 1: There exists an $l \in \mathbb{N}$ such that $\forall e \in i(E): \operatorname{co}_{\mathbb{Q}}\left(n_{e}\right) \in \frac{1}{l} \mathbb{Z}^{d}$.

Indeed, choose a transversal $s: F \rightarrow i(E)$. Then any $n_{e}$ can be written as a product $n \cdot n_{s(f)}$ for some $n \in N$ and $f \in F$. Now, $\operatorname{co}_{\mathbb{Q}}\left(n \cdot n_{s(f)}\right)$ depends polynomially on $c_{\mathbb{Q}}(n)$ and $c_{\mathbb{Q}}\left(n_{s(f)}\right)\left(\right.$ Corollary 2.3). As $c o_{\mathbb{Q}}(n) \in \frac{1}{k} \mathbb{Z}^{d}$ and there are only finitely many possibilities for $c_{\mathbb{Q}}\left(n_{s(f)}\right)$, we can conclude that the claimed $l$ really exists.

Fact 2: $\tilde{K}_{\lambda}(e) \cdot e^{-1}=\tilde{K}_{\lambda}\left(n_{e}\right) \cdot n_{e}^{-1}$.

This follows from the calculation

$$
\begin{aligned}
\tilde{K}_{\lambda}(e) \cdot e^{-1} & =\tilde{K}_{\lambda}\left(n_{e}, \varphi_{e}\right) \cdot\left(n_{e}, \varphi_{e}\right)^{-1} \\
& =\left(K_{\lambda}\left(n_{e}\right), \varphi_{e}\right)\left(\varphi_{e}^{-1}\left(n_{e}^{-1}\right), \varphi_{e}^{-1}\right) \\
& =\left(K_{\lambda}\left(n_{e}\right) \cdot n_{e}^{-1}, 1\right) \\
& =\tilde{K}_{\lambda}\left(n_{e}\right) \cdot n_{e}^{-1} .
\end{aligned}
$$

Fact 3: The map

$$
\chi:(\mathbb{Q} \backslash\{0\}) \times \mathbb{Q}^{d} \rightarrow \mathbb{Q}^{d}:(\lambda, \vec{q}) \mapsto c_{\mathbb{Q}}\left(\tilde{K}_{\lambda}\left(c_{\mathbb{Q}}^{-1}(\vec{q})\right) \cdot\left(c o_{\mathbb{Q}}^{-1}(\vec{q})\right)^{-1}\right)
$$

is a (rational) polynomial map in both $\lambda$ and $\vec{q}=\left(q_{1}, q_{2}, \ldots, q_{d}\right)$.

This follows from the fact that the expression for $K_{\lambda}$ with respect to any basis of $\mathfrak{n}_{\mathbb{Q}}$ is a polynomial in $\lambda$, and from the fact that products and inverses are also expressed by polynomial maps in the coordinates. 
Fact 4: There exists a polynomial map $\xi: \mathbb{Q} \times \mathbb{Q}^{d} \rightarrow \mathbb{Q}^{d}$ such that

$$
\forall \vec{q} \in \mathbb{Q}^{d}, \forall r \in \mathbb{Q}: \chi(1+r, \vec{q})=r \xi(r, \vec{q}) .
$$

By Fact $3, \chi(1+r, \vec{q})$ is polynomial in both $r$ and $\vec{q}$. Moreover, for $r=0$ (i.e., $\lambda=1)$, we have that

$$
\begin{aligned}
\chi(1, \vec{q}) & =c o_{\mathbb{Q}}\left(\tilde{K}_{1}\left(c o_{\mathbb{Q}}^{-1}(\vec{q})\right) \cdot\left(c o_{\mathbb{Q}}^{-1}(\vec{q})\right)^{-1}\right) \\
& =c o_{\mathbb{Q}}\left(c_{\mathbb{Q}}^{-1}(\vec{q}) \cdot\left(c_{\mathbb{Q}}^{-1}(\vec{q})\right)^{-1}\right) \\
& =c o_{\mathbb{Q}}(1) \\
& =\overrightarrow{0} .
\end{aligned}
$$

This implies that $r$ is a factor of the polynomial $\chi(1+r, \vec{q})$, or that

$$
\chi(1+r, \vec{q})=r \xi(r, \vec{q}) \text { for some polynomial } \xi(r, \vec{q}),
$$

as claimed.

Using these 4 facts, we can now finish the proof of the lemma. Let $L$ be an integer satisfying

$$
\forall r \in \mathbb{Z}, \forall \vec{q} \in \frac{1}{l} \mathbb{Z}^{d}: \quad \xi(r, \vec{q}) \in \frac{1}{L} \mathbb{Z}^{d} .
$$

(For example, one can take $L=l^{g} \cdot m$, where $g$ is the degree of the polynomial map $\xi$ and $m$ is the least common multiple of the denominators of the coefficients appearing in $\xi$.) Now, by taking $r=L \cdot k$ we find that

$$
\forall \vec{q} \in \frac{1}{l} \mathbb{Z}^{d}: \chi(1+r, \vec{q}) \in k \mathbb{Z}^{d} .
$$

This shows that

$$
\forall e \in i(E): \tilde{K}_{\lambda}(e) \cdot e^{-1} \in N,
$$

which proves (something more than) what was asked for.

Proof of Theorem [5.1] We use all the notation introduced so far. Without loss of generality, we can assume that $M=i(E) \backslash N_{\mathbb{R}}$, where

$$
i: E \rightarrow N_{\mathbb{Q}} \rtimes \operatorname{Aut}\left(N_{\mathbb{Q}}\right) \subseteq N_{\mathbb{R}} \rtimes \operatorname{Aut}\left(N_{\mathbb{R}}\right)
$$

is the rational representation of $E$ used above.

Let $\lambda>1$ be as obtained in Lemma 5.2 The map $K_{\lambda}$, seen as an automorphism of $N_{\mathbb{R}}$, is an expanding automorphism. If we look at the conjugation by $K_{\lambda}$ inside $N_{\mathbb{R}} \rtimes \operatorname{Aut}\left(N_{\mathbb{R}}\right)$, we see that

$$
\begin{aligned}
\forall e \in i(E): K_{\lambda} \cdot e \cdot K_{\lambda}^{-1} & =\left(1, K_{\lambda}\right)\left(n_{e}, \varphi_{e}\right)\left(1, K_{\lambda}^{-1}\right) \\
& =\left(K_{\lambda}\left(n_{e}\right), \varphi_{e}\right)\left(\text { recall that }\left[K_{\lambda}, \varphi_{e}\right]=1\right) . \\
& =\left(K_{\lambda}\left(n_{e}\right) \cdot n_{e}^{-1}, 1\right) \cdot\left(n_{e}, \varphi_{e}\right) \\
& =\underbrace{\tilde{K}_{\lambda}(e) \cdot e^{-1}}_{\in i(E)} \cdot e \in i(E) .
\end{aligned}
$$

This implies that $K_{\lambda}$ induces an expanding infra-nilmanifold endomorphism of $M$. 


\section{REFERENCES}

[1] Dekimpe, K. Almost-Bieberbach Groups: Affine and Polynomial Structures, volume 1639 of Lecture Notes in Math., Springer-Verlag, 1996. MR 2000b:20066

[2] Dekimpe, K. and Malfait, W. Affine structures on a class of virtually nilpotent groups. Topol. and its Applications, 1996, 73 (2), pp. 97-119. MR 97j:57060

[3] Gromov, M. Groups of polynomial growth and expanding maps. Inst. Hautes Études Sci. Publ. Math., 1981, 53, pp. 53-73. MR 83b:53041

[4] Hall, P. The Edmonton Notes on Nilpotent Groups. Queen Mary College Math. Notes, London, 1969. MR 44:316

[5] Johnson, R. W. Homogeneous Lie Algebras and Expanding Automorphisms. Proc. Amer. Math. Soc., 1975, 48 (2), pp. 292-296. MR 51:10417

[6] Kargapolov, M. and Merzljakov, J. Fundamentals of the Theory of Groups, volume 62 of Grad. Texts in Math. Springer-Verlag, 1979. MR 80k:20002

[7] Lee, H. and Lee, K. B. Expanding maps on 2-step infra-nilmanifolds. Topology Appl., 2002, 117 (1), pp. 45-58. MR 2002i:57038

[8] Lee, K. B. and Raymond, F. Rigidity of almost crystallographic groups. Contemporary Math. Amer. Math. Soc., 1985, 44, pp. 73-78. MR 87d:57026

[9] Leger, G. Derivations of Lie Algebras III. Duke Math. J., 1963, 30 (4), pp. 637-645. MR 28:3064

[10] Mal'cev, A. I. On a class of homogeneous spaces. Translations Amer. Math. Soc., 1951, 39, pp. 1-33. MR 12:589e

[11] Segal, D. Polycyclic Groups. Cambridge Tracts in Mathematics, No. 82, Cambridge University Press, 1983. MR 85h:20003

Katholieke Universiteit Leuven Campus KortriJk, Universitaire Campus, B-8500 KORTRIJK, BELGIUM

E-mail address: Karel.Dekimpe@kulak.ac.be

University of OKLahoma, Norman, OKLahoma 73019

E-mail address: kblee@math.ou.edu 